\title{
A STUDY OF AETIOLOGICAL AGENTS OF FUNGAL RHINOSINUSITIS
}

\author{
Sadarudheen Ahmed Mohammed Islah', Vanajakshi Sambasivan Sajeev²
}

${ }^{1}$ Additional Professor, Department of ENT, Government Medical College, Alappuzha. 2Junior Resident, Department of ENT, Government Medical College, Kottayam.

\begin{tabular}{l}
\hline ABSTRACT \\
\hline BACKGROUND \\
Etiological agents of fungal rhinosinusitis in our geographical area are largely unknown to us since it is not subjected to careful \\
studies. We usually depend western data for the same which can be different from that of ours. \\
Objective- To identify the aetiological agents of fungal rhinosinusitis.
\end{tabular}

\section{MATERIALS AND METHODS}

This prospective study was conducted in one hundred and ninety six patients who presented with symptoms and signs of fungal rhinosinusitis in the Department of ENT, who underwent sinus surgery and investigations including fungal culture and histopathological examination. Out of 196 cases 40 patients turned out positive for fungal organisms as evidenced by fungal culture and histopathological examination.

\section{RESULTS}

Out of the forty patients, $15(37.5 \%)$ were Aspergillus niger, 14(35\%) were Aspergillus fumigatus, 4(10\%) were Alternaria, $3(7.5 \%)$ were Mucor, $3(7.5 \%)$ were Curvularia and $1(2.5 \%)$ was Bipolaris

\section{CONCLUSION}

The etiological agents of fungal sinusitis identified in the study population were Aspergillus niger, Aspergillus fumigatus, Alternaria, Mucor, Curvularia and Bipolaris. Allergic fungal sinusitis (AFS) was the most frequently occurring fungal rhinosi nusitis disorder and Aspergillus was the most common organism isolated. Chronic invasive fungal sinusitis was exclusively seen in uncontrolled diabetics in the present study and the organism isolated were Mucor in three cases and Aspergillus niger in the other two cases.

\section{KEYWORDS}

Fungal Rhinosinusitis, Etiological Agents, Fungal Culture, Histopathological Examination.

HOW TO CITE THIS ARTICLE: Islah SAM, Sajeev VS. A study of aetiological agents of fungal rhinosinusitis. J. Evolution Med. Dent. Sci. 2017;6(3):179-184, DOI: 10.14260/Jemds/2017/44

\section{BACKGROUND \\ Fungal rhinosinusitis has been a known medical entity for several hundred years. But only in more recent times the entity has been further defined. It is one of the important health care problems and its incidence and prevalence are increasing in our population. In addition to the physical symptoms it also results in substantial functional and emotional impairment. Fungal aetiology should be suspected in all patients with chronic sinusitis.}

\section{There are Two Types of Fungal Rhinosinusitis}

1. Noninvasive fungal rhinosinusitis consists of.
a. Fungus ball.
b. Allergic fungal rhinosinusitis.

2. Invasive fungal rhinosinusitis consists of.

a. Chronic or indolent invasive fungal rhinosinusitis.

b. Acute fulminant rhinosinusitis.

c. Granulomatous invasive fungal rhinosinusitis.

Financial or Other, Competing Interest: None.

Submission 02-12-2016, Peer Review 26-12-2016,

Acceptance 02-01-2017, Published 09-01-2017.

Corresponding Author:

Dr. Sadarudheen Ahmed Mohammed Islah,

Additional Professor,

Department of ENT,

Government Medical College

Vandanam, P.O, Alappuzha.

E-mail: drsadarudeen@yahoo.com

DOI: $10.14260 /$ jemds $/ 2017 / 44$

\section{(c) (i) $(9)$}

\section{Objectives}

To find out the etiological agents of fungal rhino sinusitis.

\section{MATERIALS AND METHODS}

Study Design

Prospective study.

\section{Study Setting}

Department of ENT MCH, Kottayam.

\section{Study Subjects}

All patients attending ENT department with history suggestive of fungal rhinosinusitis.

Symptoms include purulent nasal discharge which may be blood stained, nasal obstruction, bleeding from nose, severe headache, halitosis, cacosmia, diplopia, visual impairment, sneezing and lacrimation. Signs include thick purulent nasal discharge, epistaxis, nasal crusting, granulation, ulceration, black necrotic turbinate, nasal polyps, nasal mass, swelling in the cheek, proptosis and associated third, fourth, fifth and sixth cranial nerve involvement suggestive of orbital apex syndrome.

\section{Study Duration}

$11 / 2$ years from February 2011. 


\section{Inclusion Criteria}

1. All inpatients and outpatients attending the ENT department of Kottayam medical college with symptoms and signs of fungal rhino sinusitis for one and a half years from February 2011.

2. All patients undergoing nasal surgery for fungal rhino sinusitis in the ENT department for one and a half years from February 2011.

\section{Exclusion Criteria}

1. Patients who are not willing to get involved in the study

2. Critically ill patients.

\section{Procedure}

After taking history, detailed ear, nose and throat examination including general examination was done and recorded on the proforma. Diagnostic nasal endoscopy was included in the workup.

Apart from the routine investigations, radiological investigations including $\mathrm{X}$ ray and CT scan of the nose and paranasal sinuses was done in all cases to know the sinuses involved, bony erosions and invasion of adjacent structures. Nasal discharge of the patients with history suggestive of fungal rhinosinusitis is send for fungal culture. At surgery, the allergic mucin and specimen are sent for fungal culture and histopathologic examination. For fungal culture the specimen was sent in normal saline. Although there are several culture media for fungi, the medium most commonly used in our department was the Sabouraud agar which is the general medium for cultivation of fungus.

For histopathological examination, the specimen was first treated with $10 \%$ potassium hydroxide and then stained using either Giemsa, PAS, Gomori methenamine silver or Calcofluor white.

The reports of fungal culture and histopathology are collected and analysed by statistical methods and the prevalence of the organisms in the study group assessed.

\section{RESULTS}

The study was conducted for a period of one and a half years in 196 cases of sinonasal diseases who presented with signs and symptoms suggestive of fungal rhinosinusitis. 40 patients turned out to be positive for fungal organisms as evidenced by clinical, radiological and laboratory findings.

\section{Incidence}

The incidence of fungal sinusitis among 196 cases of sinonasal diseases was $20.40 \%$, ie. 40 out of 196 cases.

\section{Age and Sex Distribution}

The age incidence showed a wide variation. The youngest patient was a 11 year old male. The oldest was a 75 year old male. In the study, there were 22 male and 18 female patients. There was a equal male and female patients in the younger age group, ie. (10-30 yrs.), 4 males and 4 females out of the 8 cases. Between 31-80 yrs. there was a male predominance. Out of the 32 cases, 19 (59.37\%) males and 13 (40.63\%) females.

Overall there was a male predominance, that is 22 out of 40 (55\%) cases and females 18 cases out of 40 (45\%).

\begin{tabular}{|c|c|c|}
\hline Age & Cases & Percentage \\
\hline $0-10$ yrs. & 0 & 0 \\
\hline $10-20$ yrs. & 4 & $10 \%$ \\
\hline $21-30$ yrs. & 4 & $10 \%$ \\
\hline $31-40$ yrs. & 9 & $22.5 \%$ \\
\hline $41-50$ yrs. & 10 & $25 \%$ \\
\hline $51-60$ yrs. & 7 & $17.5 \%$ \\
\hline $61-70$ yrs. & 5 & $12.5 \%$ \\
\hline$>70$ yrs. & 1 & $2.5 \%$ \\
\hline \multicolumn{3}{|c|}{ Table 1 } \\
\hline
\end{tabular}

\section{Age Group Incidence}

Socioeconomic status- $45 \%$ cases belonged to low class, $30 \%$ cases were of the middle class and $25 \%$ were of the high-class group.

\section{Associated Conditions}

Out of the 40 cases 17 had associated diabetes mellitus (42.5\%), 22 patients had nasal allergy (55\%),11 patients had bronchial asthma (27.5\%).

\section{Symptoms}

Patients presented with a variety of symptoms. Nasal obstruction and nasal discharge were the predominant symptoms. Other complaints include nasal bleed, headache, anosmia, proptosis and sneezing.

\section{Symptoms}

\begin{tabular}{|c|c|c|}
\hline Symptoms & Cases & Percentage \\
\hline Nasal obstruction & 38 & $95 \%$ \\
\hline Nasal discharge & 34 & $85 \%$ \\
\hline Epistaxis & 13 & $32.5 \%$ \\
\hline Headache & 3 & $7.5 \%$ \\
\hline Anosmia & 1 & $2.5 \%$ \\
\hline Proptosis & 3 & $7.5 \%$ \\
\hline Sneezing & 1 & $2,5 \%$ \\
\hline \multicolumn{2}{|c}{ Table 2 } \\
\hline
\end{tabular}

\section{Habits}

8 patients (20\%) had a history of chronic smoking. 3 cases (7.5\%) gave a history of alcoholism.

\section{Clinical Examination}

Out of total 40 positive cases of fungal rhinosinusitis 34 were allergic fungal rhinosinusitis. Out of the 34 cases of AFRS, 21 had sinonasal polyposis on clinical examination (52.5\%). Out of 40 cases 9 cases had significant deviation of nasal septum. (22.5\%). 12 patients had middle turbinate hypertrophy (30\%) 5 patients had inferior turbinate hypertrophy $(12.5 \%)$ 3 patients had proptosis (7.5\%).

\section{Investigations}

Differential blood count showed raised eosinophil count in 19 cases out of 34 cases of AFS (55.88\%) cases. Blood sugar was raised in 17 out of the 40 cases. There were 5 cases of chronic invasive fungal sinusitis in the study group. Blood sugar was raised in all the 5 cases of chronic invasive fungal sinusitis.

\section{Radiological Findings}

X-ray and CT scan of the nose and paranasal sinuses were 
taken in all patients. 8 patients (20\%) had unilateral involvement and 32 cases (80\%) had bilateral involvement Heterogenous areas of signal intensity or double intensity sign was seen in all cases. Erosion of bony walls was seen in 4 cases $(10 \%)$. Maxillary sinus was the most common sinus involved.

\begin{tabular}{|c|c|c|}
\hline Sinuses Involved & Number & Percentage \\
\hline Unilateral maxillary & 3 & $7.50 \%$ \\
\hline Bilateral maxillary & 2 & $5 \%$ \\
\hline Unilateral maxillary+ ethmoid & 1 & $2.50 \%$ \\
\hline Bilateral maxillary + ethmoid & 14 & $35 \%$ \\
\hline Bilateral maxillary + ethmoid+ frontal & 6 & $15 \%$ \\
\hline $\begin{array}{l}\text { Unilateral maxillary+ ethmoid+ } \\
\text { frontal+ sphenoid }\end{array}$ & 1 & $2.50 \%$ \\
\hline $\begin{array}{l}\text { Bilateral maxillary + ethmoid }+ \\
\text { frontal+ sphenoid }\end{array}$ & 2 & $5 \%$ \\
\hline Unilateral + ethmoid + frontal & 1 & $2.50 \%$ \\
\hline Bilateral maxillary + frontal & 5 & $12.50 \%$ \\
\hline Unilateral maxillary+ frontal & 1 & $2.50 \%$ \\
\hline Bilateral ethmoid + frontal & 1 & $2.50 \%$ \\
\hline Unilateral maxillary+ sphenoid & 1 & $2.50 \%$ \\
\hline $\begin{array}{c}\text { Bilateral maxillary +ethmoid+ } \\
\text { sphenoid } \\
\end{array}$ & 2 & $5 \%$ \\
\hline \multicolumn{3}{|c|}{ Table 3. Radiological Findings } \\
\hline
\end{tabular}

\section{Histopathological Examination}

Allergic mucin was positive for fungal hyphae in 15 cases out of 34 cases of AFS (44.11\%). Tissue invasion was seen in cases of chronic invasive fungal sinusitis on histopathological examination.

\section{Fungal Culture}

Out of 40 case 29 were positive for fungal organisms after 6 weeks. 11 cases out of 40 patients were negative for fungal organisms on fungal culture. Positive cases were $72.5 \%$ and negative cases were $27.5 \%$.

\begin{tabular}{|c|c|c|}
\hline Cases & Cases & Percentage \\
\hline Negative cases & 11 & $27.5 \%$ \\
\hline Positive cases & 29 & $72.5 \%$ \\
\hline \multicolumn{2}{|c|}{ Table 4. Fungal culture } \\
\hline
\end{tabular}

\begin{tabular}{|c|c|c|}
\hline Species Identified & $\begin{array}{c}\text { Histopathological } \\
\text { Examination }\end{array}$ & $\begin{array}{c}\text { Fungal } \\
\text { Culture }\end{array}$ \\
\hline Aspergillus fumigatus & 6 & 12 \\
\hline Aspergillus niger & 4 & 10 \\
\hline Mucor & 1 & 2 \\
\hline Alternaria & 2 & 2 \\
\hline Curvularia & 3 & 2 \\
\hline Bipolaris & 0 & 1 \\
\hline Total & $\mathbf{1 6}$ & $\mathbf{2 9}$ \\
\hline Table 5. Species identified both in Culture and \\
Histopathology \\
\hline
\end{tabular}

In 5 cases both histopathology and fungal culture gave positive results.

\begin{tabular}{|c|c|c|}
\hline Species Identified & Number of Cases & Percentage \\
\hline Aspergillus niger & 15 & $37.5 \%$ \\
\hline Aspergillus fumigatus & 14 & $35 \%$ \\
\hline Alternaria & 4 & $10 \%$ \\
\hline Curvularia & 3 & $7.5 \%$ \\
\hline Mucor & 3 & $7.5 \%$ \\
\hline Bipolaris & 1 & $2.5 \%$ \\
\hline \multicolumn{3}{|c|}{ Table 6. Final species identified } \\
\hline
\end{tabular}

\section{Diagnosis}

The types of fungal sinusitis identified were- allergic fungal sinusitis 34 cases (85\%), chronic invasive fungal sinusitis 5 cases $(12.5 \%)$, fungal ball 1 case $(2.5 \%)$. The organisms identified were Aspergillus niger 15 cases (37.5\%), Aspergillus fumigatus 14 cases (35\%), Alternaria 4 cases (10\%), Curvularia 3 cases (7.5\%), Mucor 3 cases $(7.5 \%)$, Bipolaris 1 case $(2.5 \%)$.

\section{Type of Sinusitis}

Out of the 40 cases in the study group, 34(85\%) cases were that of AFRS and $5(12.5 \%)$ were that of CIFS cases and 1 case $(2.5 \%)$ was of fungal ball.

\begin{tabular}{|c|c|c|}
\hline Type of Sinusitis & Number & Percentage \\
\hline Allergic fungal sinusitis & 34 & $85 \%$ \\
\hline Chronic invasive fungal sinusitis & 5 & $12.5 \%$ \\
\hline Fungal ball & 1 & $2.5 \%$ \\
\hline Acute invasive fungal sinusitis & 0 & $0 \%$ \\
\hline \multicolumn{3}{|c|}{ Table 7. Types of sinusitis } \\
\hline
\end{tabular}

\section{DISCUSSION}

\section{Incidence}

Among the 196 cases with history suggestive of fungal rhinosinusitis 40 cases had fungal aetiology, ie (20.4\%) cases. In Grigouriu ${ }^{1}$ et al's study, 81 out of 600 cases were found to have fungal aetiology, ie. an incidence of $13.5 \%$. Al - Dousary in a retrospective study of 406 cases of chronic sinusitis gives a $16.9 \%$ incidence of fungal sinusitis.

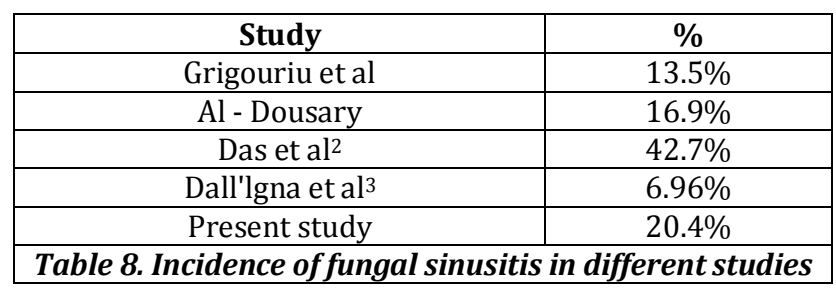

Of the 196 cases with history suggestive of fungal rhinosinusitis -34 cases had clinical, radiological and laboratory features suggestive of allergic fungal sinusitis, ie. an incidence of $17.34 \%$. Study by Katzenstein ${ }^{4}$ et al gives a 5 to $10 \%$ incidence of AFS.

\begin{tabular}{|c|c|}
\hline Study & $\mathbf{\%}$ \\
\hline Katzenstein et al & $10 \%$ \\
\hline Collins et al & $9 \%$ \\
\hline Ponikauetal & $93 \%$ \\
\hline Present study & $17.34 \%$ \\
\hline Table 9. Incidence of AFS in different studies \\
\hline
\end{tabular}


Ponikau ${ }^{5}$ et al in 1999, diagnosed AFS in 94 out of 101 consecutive patients (93\%), underwent endoscopic sinus surgery for chronic rhinosinusitis and suggested the inadequacy of the methods generally used to identify the fungi, is responsible for the low incidence of AFS in previous reports.

The incidence of AFS also appears to be influenced by geographic factors. Most areas reporting high incidence of AFS are located in temperate regions with relatively high humidity.

There was 1 case of fungal ball and 5 cases of chronic invasive fungal sinusitis in the study group.

\section{Age and Sex Characteristics}

In present study, the age varied from 11-75 years. The maximum age incidence was seen in age group 41-50 yrs. The mean age in the present study was 43.38 years. This represents the economically productive age group of community and reflects the probability of occupational exposure to fungus.

In the present study there were $22(55 \%)$ males and 18 $(45 \%)$ females. In younger age group (ll-30 yrs.) there were equal male and female predominance and in older age group (31-75 yrs.) there was slight male predominance.

\section{Socioeconomic Status}

In the study $45 \%$ belonged to poor socioeconomic class. $25 \%$ belonged to high socioeconomic class and 30\% belonged to middle class. A high incidence of $32 \%$ was noticed among farmers which could probably due to exposure to fungal antigen from work site. In study by Kalimullah, Thahim ${ }^{6}$ et al $80 \%$ belonged to low socioeconomic class.

\section{History of Allergic Rhinitis}

$22(64.70 \%)$ patients had history of allergic rhinitis out of the 34 cases of allergic fungal rhinosinusitis. According to Ferguson there was an $84 \%$ incidence of allergic rhinitis in AFS patients. In Kalimullah, Thahim et al's study of 20 cases of AFS, all patients were having history of allergic rhinitis. This results points out, fungus to be an allergen rather than a pathogen.

\section{Bronchial asthma}

11 cases (32.35\%) out of 34 AFS patients had bronchial asthma. In a study by Rishi $\mathrm{Pal}^{7}$ et al reported a $25 \%$ incidence of bronchial asthma in AFS patients. A $33.8 \%$ incidence of bronchial asthma was reported by Celso Dalligna et al. In Katzenstein study 11 cases of AFS out of 15 cases had bronchial asthma, (73.3\%).

AFS has been suggested to be the upper airway manifestation of a similar process occurring in the lower airways of atopic asthmatic patients, which is now termed allergic bronchopulmonary aspergillosis (ABPA). McCarthy and Pepys have reported that $10 \%$ of patients with ABPA produce nasal plugs that are similar to the airway casts that are present in the bronchi of patients with ABPA. Safirstein has reported that nasal discharge containing airway casts improved when a patient with ABPA was treated with corticosteroids. The hypothesis that AFS and ABPA share immune-pathogenic mechanisms is further supported by the observation that the distinctive, eosinophil-rich, laminated, allergic mucin is the substrate for the mucous plugs that are common to both conditions.

No patients had aspirin sensitivity. A $25 \%$ incidence of aspirin sensitivity in AFS patients is reported by Bee-See Goh et al A higher incidence of eosinophilic mucin sinusitis without fungus is noticed among aspirin sensitive AFS patients.

\section{Diabetes Mellitus}

All five cases $(100 \%)$ of chronic invasive fungal sinusitis had uncontrolled diabetes. All the 5 patients with chronic invasive fungal sinusitis had uncontrolled diabetes with fasting blood sugar in the range of $130 \mathrm{mg} \%$ to $150 \mathrm{mg} \%$ and post prandial blood sugar in the range of $210 \mathrm{mg} \%$ to $246 \mathrm{mg} \%$. These patients were treated with injection Human Mixtard and Metformin tablets. Over all incidence of diabetes in the present study was 17 cases out of 40 (42.5\%). In the study of Michael et al $62 \%$ cases of acute invasive fungal sinusitis were diabetic patients. In the study of Chackrabarthyet $\mathrm{al}^{8}$ fulminant fungal sinusitis occurred in patients with diabetes, malignancy, burns, trauma, and bone marrow transplantation. In a study by Parikh ${ }^{9}$ et al out of 45 cases of invasive fungal sinusitis, only 10 had diabetes mellitus.

Other immunosuppressive conditions predisposing invasive fungal sinusitis like AIDS, leukaemia, agranulocytosis, haematological diseases and patients on long term corticosteroids, broad spectrum antibiotics, cytotoxic drugs and on immunosuppressive drugs after organ transplant were not encountered in present study.

\section{Symptoms}

Nasal obstruction and nasal discharge were the most common presenting complaints of AFS in the study group. In Kalimullah et al's study nasal obstruction wasreported in $100 \%$ cases and nasal discharge in $90 \%$ cases. Headache was reported by 3 patients, (7.5\%) and in this study headache appears a less predominant symptom of AFS comparing bacterial sinusitis. Other minor complaints included nasal bleed and anosmia proptosis and sneezing. According to Galli et al, the predominant symptom was facial pain, followed by nasal obstruction.

\section{Clinical Examination}

21 out of 40 cases (52.5\%)had sinonasal polyposis on clinical examination. In Katzenstein series of 15 cases, 14 had polyps and in Ence ${ }^{10}$ et al's study $100 \%$ patients had nasal poylposis. In AL-Dousary's study 56 out of 59 cases had polyposis, ie. 94.9\%.

Other examination findings include, significant septal deviation ( 9 cases had significant deviation of nasal septum 22.5\% cases). 12 patients had Middle turbinate hypertrophy (30\% cases). 5 patients had inferior turbinate hypertrophy in (12.5\% cases). 3 cases had proptosis 7.5\%. Kalimullah et al reported $20 \%$ incidence of proptosis, $20 \%$ incidence of telecanthus and facial dysmorphism of $20 \%$. Facial dysmorphism and telecanthus are significant in children.

\section{Investigations}

Differential blood count showed raised eosinophil count in 19 cases out of 34 cases of allergic fungal sinusitis (55.88\%). Blood sugar was raised in 5 cases of chronic invasive fungal sinusitis and in 17 cases out of total 40 cases. Ravikumar'11 et 
al in a study gave a $60 \%$ incidence of raised eosinophil count in AFS. Eosinophilia signifies the allergic predisposition of the patient.

\section{CT Scan of Nose and Paranasal Sinuses}

CT scan was taken in all cases. Computed Tomography of the paranasal sinuses has improved the visualisation of paranasal sinus anatomy and has allowed greater accuracy in evaluating paranasal sinus disease. It evaluates the osteomeatal complex anatomy which is not possible with plain radiographs. The area of scanning was defined to include the region from roof of frontal sinus up to the hard palate. The sections were taken with slice thickness of $5 \mathrm{~mm}$. The sinuses involved, bony expansion, erosion and extension into adjacent structures were identified. The triad of nasal polyps, characteristic CT scan findings and specific immunoglobulin $\mathrm{E}$ titers has been shown to have a sensitivity of $70 \%$ and a specificity of $100 \%$ for the preoperative diagnosis of allergic fungal sinusitis.

The expanding allergic mucin may cause thinning and erosion of the bone. Bony remodelling or erosion is thought to be secondary to pressure atrophy or the release of inflammatory mediators, that dissolve bone, and is not due to fungal invasion.

In MRI, the corresponding areas of hyper attenuation on CT scan are seen as areas of hypo intensity on $\mathrm{Tl}$ weighted images and signal void on T2 weighted images. The combined CT scan and MRI findings provide a radiographic appearance that is highly specific for allergic fungal sinusitis (AFS). Single sinus involvement was seen in 3 cases, ie $7.5 \%$. Multi sinus involvement was seen in 37 cases, (92.5\%) in the present study. Al- Dousary in a study of 59 cases reported a $100 \%$ incidence of multi sinus disease.

\section{Fungal Culture}

Of the 34 AFS cases, 29 cases gave positive fungal culture, ie $85.29 \%$. Fungal cultures were positive in, 70 to $80 \%$ of patients diagnosed with AFS (Kupferberg). The Mayo Clinic reports virtually $100 \%$ positive fungal cultures on all patients, irrespective of pathology. This points out the possibility of false positives if one relies on fungal cultures, given the ubiquity of fungal spores. Why all patients with histopathologic evidence of AFS do not grow fungal cultures has been attributed to over homogenisation of the specimen prior to plating, which disrupts the fragile hyphal walls, a delay in incubating the culture, and inappropriate culture techniques to separate fungi from the thick mucin in which they grow.

Aspergillus was the main aetiological fungus in fungal sinusitis in the present study. There were 15 cases of Aspergillus niger (37.5\%) and 14 cases of Aspergillus fumigates $(35 \%)$. The next common fungi isolated were Alternaria 4 (10\%), Curvularia, 3 (7.5\%), Mucor, 3 (7.5\%) and bipolaris $1(2.5 \%)$.

According to Manning ${ }^{12}$ et al dematiaceous fungi were the most common isolates based on culture data and account for $84 \%$ of the total positive cultures. The most common fungi were Bipolaris species, followed Curvularia, Alternaria, and Exserohilum. Aspergillus species accounted for $13 \%$ of all fungal cultures. This variation may be due to the geographic characteristics or techniques in fungal culture.

\begin{tabular}{|c|c|}
\hline Fungus Species & No. of Cases \\
\hline A. fumigatus & 31 \\
\hline A. fumigatus + Penicillium & 3 \\
\hline A. fumigatus + Cladosporium & 3 \\
\hline A. fumigatus + Fusarium & 1 \\
\hline A. fumigatus + A niger & 1 \\
\hline Cladosporium & 4 \\
\hline Penicillium & 1 \\
\hline Cladosporium + Fusarium & 1 \\
\hline No fungus on culture & 3 \\
\hline Total & 48 \\
\hline \multicolumn{2}{|c|}{$\begin{array}{l}\text { Table 10. Study of H. Stammberger13 University } \\
\text { ENT clinic, Graz, Australia }\end{array}$} \\
\hline
\end{tabular}

In the study of GRANNILE14 et al, $67 \%$ cases had positive fungal culture. Demateaceous fungi were the most common organisms.

In the study of RAJIV.C.MICHAEL, CMC Vellore $63 \%$ had AFRS and $34 \%$ had IFS. Aspergillus species was the most common organism in AFRS.

In the present study one cases of fungal ball grew Aspergillus fumigatus in culture. Out of the 5 cases of chronic invasive fungal sinusitis. Mucor were the aetiological agents in three cases and Aspergillus fumigates were the etiological agent in rest of the 2 cases of chronic invasive fungal sinusitis. In most of the studies the common type of sinusitis identified were allergic fungal sinusitis and the most common organisms identified were aspergillus species. This is similar to the present study. But the common organism identified in chronic invasive fungal rhinosinusitis in the present study is Mucor which is different from that of other studies.

\section{Histopathological Examination}

On histopathological examination of the biopsy specimen in 16 cases, fungal organisms were identified, out of which 15 cases were that of allergic fungal sinusitis and one case was that of chronic invasive fungal sinusitis. Under the microscope, allergic mucin reveals necrotic eosinophils, frequently in wave like concentric layers. On higher power, Charcot-Leyden crystals are seen. They are thought to be a product of eosinophil degranulation. Diagnostically most important is the finding of hyphal fragments scattered throughout the eosinophilic mucin. Special stains for fungi such as Gomori methenamine-silver are frequently required to see these hyphae, although they may occasionally be appreciated on haematoxylin and eosin stain. One cannot differentiate among the many fungi that cause AFS based on histopathological appearance.

Most cases are not caused by the Aspergillus species, in contradistinction to the early assumptions by Millar15 et al and Katzenstein et al, who initially designated AFS as allergic Aspergillus sinusitis. Rather, several types of fungi, including Aspergillus, are responsible. Ultimate attribution to a particular fungal species depends on fungal culture results. Preoperative steroids may sometimes obscure the diagnosis, as detailed in one case of a man with characteristic clinical findings for AFS treated with 5 days of prednisone $60 \mathrm{mg}$ daily preoperatively. Fungal hyphae were present in 15 patients (44.17\%). with AFS. 4 of them had bronchial asthma and no one had aspirin sensitivity (Samter's triad). Occasionally, patients with clinical characteristics of AFS are 
noted to have characteristic eosinophilic mucin but no evidence of hyphae, despite special stains. Alphin ${ }^{16}$ et al reported on 13 patients with this polypoid histopathology and eosinophilic mucin but absent fungi and negative fungal cultures. Interestingly, all patients in both reports had moderate to severe asthma and were aspirin sensitive. This group of patients, with eosinophilic mucin sinusitis without hyphae and severe asthma that is often aspirin sensitive, represent a group of patients with a different pathophysiologic basis for their sinus disease than patients with AFS. The most probable cause of AFS as already stated is a hypersensitivity reaction to fungi. Those with eosinophilic mucin sinusitis without fungus probably have a systemic immunologic abnormality, which would account for their asthma and the involvement of all sinuses.

Tissue invasion was typically absent in all cases of AFS. 1 cases of chronic invasive fungal sinusitis showed evidence of tissue invasion on histopathology and was caused by Mucor.

\section{CONCLUSION}

The following conclusions were made in the study.

1. The etiological agents of fungal sinusitis identified in the study population were Aspergillus Niger, Aspergillus Fumigatus, Alternaria, Mucor, Curvularia and Bipolaris.

2. Allergic fungal sinusitis (AFS) was the most frequently occurring fungal rhinosinusitis disorder and Aspergillus was the most common organism isolated.

3. Chronic invasive fungal sinusitis was exclusively seen in uncontrolled diabetics in the present study and the organism isolated were Mucor in three cases and Aspergillus Niger in the other two cases.

4. Majority of AFS patients belonged to low socioeconomic class.

5. AFS usually coexisted with allergic rhinitis possibly due to the fact that fungus acts as an allergen rather than a pathogen. A significant number of patients with AFS had associated bronchial asthma.

6. Nasal obstruction and nasal discharge were the most common presenting complaints.

7. Headache was a less common symptom of AFS, comparing bacterial sinusitis

8. In 19 cases of AFS there were raised eosinophil count, reflecting the allergic pathophysiology of the disease.

9. AFS showed multi sinus disease and bilateral involvement in majority of patients.

10. Maxillary sinus was the most commonly involved sinus. Unilateral involvement of the maxillary sinus was seen in Fungal ball case and Aspergillus fumigatus was the organism identified. Fungal culture and histopathological examination of allergic mucin were specific for diagnosis of AFS.

\section{REFERENCES}

[1] Grigoriu D, Bambule J, Delacretaz J, et al. La sinusite maxillaire fongique. Dermatologica 1979; 159 (Suppl1):180-6.

[2] Das A, Bal A, Chakrabarti A, et al. Spectrum of fungal rhinosinusitis; histopathologists perspective. Histopathology 2009;54(7):854-9.

[3] Dall'Igna C, Palombini BC, Anselmi F, et al. Fungal rhinosinusitis in patients with chronic sinusal disease. Braz J Otorhinolaryngol 2005;71(6):712-20.

[4] Katzenstein AL, Sale SR, Greenberger PA. Allergic aspergillus sinusitis: a newly recognized form of sinusitis. J Allergy Clin Immunol 1983;72(1):89-93.

[5] Ponikau JU, Sherris DA, Kern EB, et al. The diagnosis and incidence of allergic fungal sinusitis. Mayo clinic proceedings 1999;74(9):877-84.

[6] Kalimullah T, Jawaid MA, Saleem MM. Presentation and management of allergic fungal sinusitis. JCPSP 2007;17(1):23-7.

[7] Gupta RP, Bahadur S, Thakar A, et al. Management protocols of allergic fungal sinusitis. Indian Journal of Otolaryngology and Head \& Neck Surgery 2007;59(1):35-40.

[8] Chakarabarthi A, Sharma SC. Paranasal sinus mycoses. Indian journal of chest disease and allied sciences 2000;42:292-304.

[9] Parikh SL, Venkatraman G, Delgaudio JM. Invasive fungal sinusitis: a 15-year review from a single institution. Am J Rhinol 2004;18(2):75-81.

[10] Ence BK, Gourley DS, Jorgensen NL, et al. Allergic fungal sinusitis. Am J Rhinol 1990;4:169-78.

[11] Ravikumar A, Mohanty S, Vatsanath RP, et al. Allergic fungal sinusitis a clinico-pathological study. Indian Journal of Otolaryngology and Head and Neck surgery 2004;56(4):317-20.

[12] Manning SC, Holman M. Further evidence for allergic pathophysiology in allergic fungal sinusitis. Laryngoscope 1998;108(10):1485-96.

[13] Stammberger H. Endoscopic surgery for mycotic and chronic recurring sinusitis. Ann Otol Rhinol Laryngol 1985;119:1-11.

[14] Granville L, Chirala M, Cernoch P, et al. Fungal sinusitis: histologic spectrum and correlation with culture. Human Pathol 2004;35(4):474-81.

[15] Millar JW, Johnston A, Lamb D. Allergic aspergillosis of the maxillary sinuses. Thorax 1981;36:710.

[16] Allphin AL, Strauss M, Abdul-Karim FW. Allergic fungal sinusitis: problems in diagnosis and treatment. Laryngoscope 1991;101(8):815-20. 